\title{
CAPACITAÇÃO DE IDOSOS NA PREVENÇÃO DE QUEDAS DOMICILIARES UTILIZANDO TECNOLOGIAS DA INFORMAÇÃO E COMUNICAÇÃO
}

\author{
ELDERLY TRAINING FOR PREVENTION OF HOME FALLS USING INFORMATION AND \\ COMMUNICATION TECHNOLOGIES
}

\author{
Karis de Campos ${ }^{a}$, Mariana Alves dos Santos ${ }^{b}$, Nicolli Macedo Barros ${ }^{c}$, Thaís Marques \\ Simionato ${ }^{\mathrm{d}}$, Jayse Gimenez Pereira Brandão ${ }^{\mathrm{e}}$, Ana Paula Mansano Cunha Ramos ${ }^{\mathrm{f}}$ \\ akarisdecampos@yahoo.com.br, bmari_88_454@hotmail.com, cninicolli2@hotmail.com, dthais.x3@hotmail.com, ejayse123@hotmail.com, \\ fapmansano@yahoo.com.br \\ Faculdade de Medicina da Universidade de Mogi das Cruzes - Mogi das Cruzes (SP), Brasil
}

Data de recebimento do artigo: 10/11/2016

Data de aceite do artigo: 15/02/2017

\section{RESUMO}

Introduçáo: Quedas são consideradas um problema de saúde pública devido a sua elevada morbimortalidade, sendo uma das principais causas de óbito acidental em indivíduos acima de 65 anos. Também acarreta perda de capacitação entre os idosos, reduzindo a qualidade de vida. Objetivos: Elaborar um programa de capacitação para idosos sobre queda domiciliar através das Tecnologias da Informação e Comunicação (TICs), e posterior disseminaçáo do conhecimento adquirido aos seus pares; avaliação da eficácia do programa de capacitação. Metodologia: O estudo foi desenvolvido por cinco alunas da Liga Acadêmica de Geriatria da Faculdade de Medicina da Universidade de Mogi das Cruzes com a Universidade Aberta à Integração (Unai) em Mogi das Cruzes/SP. Participaram 11 alunos da Unai, de forma voluntária, de ambos os sexos, com 60 anos ou mais. As atividades foram divididas em cinco etapas utilizando as TICs, além da aplicação de um questionário pré e pós-capacitaçáo sobre o tema a esses participantes. Os resultados foram analisados de forma qualitativa e de estatística descritiva. Resultados: Os idosos possuíam um bom conhecimento prévio sobre os assuntos relacionados a quedas. A maioria das questóes era conhecida, ao passo que manteve-se um alto índice de acerto pré e pós-capacitação. O conteúdo transmitido foi exposto pelos participantes aos demais acadêmicos da Unai por meio de aula expositiva e teatro. Conclusáo: Observou-se a aceitaçáo e o interesse dos idosos em relação às tecnologias utilizadas durante a capacitação e a apresentação aos seus pares, o que contribuiu para o aumento do conhecimento sobre quedas domiciliares, constatado no questionário pós-capacitação.

Palavras-chave: Telemedicina; envelhecimento; comunicação; geriatria; educação em saúde.

\section{ABSTRACT}

Introduction: Falls are considered a public health problem due to their high morbidity and mortality, being one of the main causes of accidental death in individuals over 65 years. They also entail loss of capacity among the elderly, reducing their quality of life. Objectives: To elaborate for the elderly a training program on home falls using Information and Communication Technologies (ICTs), subsequently disseminating the acquired knowledge to their peers; and to evaluate the effectiveness of the training program. Methodology: The study was developed by five students of the Academic League of Geriatrics of the Faculty of Medicine of the University of Mogi das Cruzes with the University Open to Integration (Unai) in Mogi das Cruzes (SP), Brazil. Eleven Unai students of both sexes, aged 60 and over, participated voluntarily of this study. The activities were divided into five stages using ICTs in addition to the application of a pre and a post-qualification questionnaire on the subject. The results were analyzed qualitatively and in a descriptive statistics way. Results: Elderly people had a good prior knowledge of falls related subjects. Most of the questions were known, and the high pre and post-qualification hit rate was maintained. The transmitted content was exposed by the participants to the other Unai academics through lectures and theater. Conclusion: There was acceptance and interest of the elderly regarding the technologies used during the training and the presentation to their peers, which contributed to the increase in knowledge about home falls, verified in the post-qualification questionnaire.

Keywords: Telemedicine; aging; communication; geriatrics; health education. 


\section{Introdução}

O processo de envelhecimento populacional é um fenômeno analisado em todo o mundo, especialmente nos países em desenvolvimento ${ }^{1}$. No Brasil a expectativa de vida é de 74,6 anos ${ }^{2}$. De acordo com as projeçóes para 2050, o número de idosos será de um quinto da população nos países em desenvolvimento e de um terço em países desenvolvidos ${ }^{3}$.

Em vista deste processo de envelhecimento da população, é fundamental, que haja investimentos na área da saúde, com capacitação de profissionais para atuar nessa área ${ }^{4,5,6}$

A geriatria é, então, uma especialidade médica centenária responsável pela saúde e pelas doenças dos idosos. Os cuidados com quadros agudos, crônicos e de reabilitação ultrapassam a medicina curativa e abrangem aspectos paliativos e preventivos relacionados às condiçôes físicas, funcionais e sociais ${ }^{7}$. Dessa forma, a assistência ao idoso deve ser realizada de forma holística, considerando o meio social em que vivem, assim como o contexto familiar, político e histórico, melhorando a qualidade de vida ${ }^{6,8}$.

Durante esta fase da vida podem surgir as síndromes geriátricas, os chamados "gigantes da geriatria", que incluem insuficiência cerebral, instabilidade de postura e quedas, ocorrências iatrogênicas, imobilidade e incontinência ${ }^{9}$. Ao restringir o tratamento da doença e náo a visão integral do idoso ocorre uma das principais causas de iatrogenia que leva aos "gigantes da geriatria", promovendo a perda da autonomia e da independência ${ }^{10}$.

O envelhecimento é um processo dinâmico gradativo, o qual resulta no declínio das funções fisiológicas e morfológicas, tornando o organismo mais susceptível a agressóes intrínsecas e extrínsecas. Apesar de não representar um sinônimo de incapacidade ou dependência, com a progressão do evento a probabilidade de ocorrer lesôes causadas por acidentes, como as quedas, aumenta ${ }^{11}$.

Queda, portanto, tem como definição um evento não intencional que promove uma mudança de posição do corpo para um nível inferior à sua posição inicial, sendo o indivíduo incapaz de correção em tempo hábil, determinado por diversos fatores que comprometem o equilíbrio ${ }^{1}$. As circunstâncias podem ser de ordem intrínseca, extrínseca, farmacológica ou social degenerativa ${ }^{12}$.

Dentre os fatores intrínsecos que são relacionados ao processo natural de envelhecimento, podem ser mencionados: alteração da marcha e estabilidade, neuropatia periférica, depressão, debilidade muscular, deterioração cognitiva, alteração na realização das atividades de vida diárias devido ao sedentarismo e modificação da visão (distúrbios de percepção ambiental, por exemplo) e audição ${ }^{13}$. Há uma relação diretamente proporcional entre o comprometimento cognitivo e a frequência de quedas ${ }^{14}$. Como outra causa intrínseca, tem-se o uso de medicamentos de uso contínuo que podem ter como consequências processos patológicos crônico-degenerativos ${ }^{12}$.

Já as causas extrínsecas estão ligadas às situaçóes cotidianas e ambientais, destacando o ambiente domiciliar como o local com maior fator de risco para possíveis acidentes (20-58\%), sendo que superfícies irregulares, superfícies molhadas/escorregadias, objetos/tapetes soltos e desníveis no chão/problemas com degraus são as causas mais prevalentes ${ }^{15,16}$.

As quedas são consideradas um problema de saúde pública devido à elevada ocorrência de mortalidade e morbidade, além da perda de capacitaçáo entre os idosos e decréscimo da qualidade de vida, uma vez que o idoso pode adquirir receio ao andar, perda da autonomia com consequente aumento de dependência dos familiares e cuidadores $^{17}$. Estudos apontam que $30 \%$ dos idosos no Brasil caem pelo menos uma vez ao ano, tendo a maior parte desse grupo (51\%) mais de 85 anos $^{18}$. Em sua maioria as quedas ocasionam lesões, tornando-se uma das principais causas de óbito acidental em indivíduos acima de 65 anos $^{19}$. As principais lesóes ocasionadas pelas quedas são as fraturas, as quais possuem um maior grau de incidência em idosos do sexo feminino ${ }^{20}$. No período entre 1997-2010, dos 8.142.342 óbitos no Brasil entre pessoas com 60 anos de idade ou mais, $50.348(0,61 \%)$ foram óbitos por quedas, sendo que $50,6 \%$ destes ocorreram entre a população com 80 anos ou mais ${ }^{21}$.

Apesar das graves consequências, sabe-se que grande parte das quedas poderiam ser evitadas com simples prevençóes, tais como: criar ambientes seguros com solo antiderrapante, iluminação e mobília apropriadas, calçados adequados, degraus de escadas evidentes e barras de apoio em locais estratégicos para auxilio da locomoção ${ }^{15}$. Outras medidas preventivas são: revisão da medicação, promoção da saúde (atividades físicas condizentes com as limitaçōes individuais) e segurança (dentro e fora do domicílio) ${ }^{22}$.

Diante desse contexto, há a necessidade de meios educacionais para passar não apenas a essa população, mas também à populaçáo geral informaçóes de como prevenir situaçóes que possam trazer morbidades, como as consequências da queda. Uma das formas é a utilização da Tecnologia da Informação e Comunicação (TIC), que tem como finalidade informar e disseminar o conhecimento por meio de estratégias de educaçáo, podendo ser aplicada a pessoas da população em geral. Com maior informação, educação em saúde e execução de medidas públicas preventivas, há uma melhora na qualidade de vida do idoso além da diminuição dos gastos com atenção secundária e terciária ${ }^{12}$.

O projeto Jovem Doutor é uma iniciativa da Disciplina de Telemedicina da Faculdade de Medicina 
da Universidade de São Paulo (FMUSP) em que há utilização das TICs para promover educação em saú$\mathrm{de}^{23}$, que vem justamente ao encontro dessa realidade. Já houve ediçóes nas cidades de São Paulo e Tatuíi ${ }^{24}$ e em parceria com o Departamento de Fonoaudiologia de Bauru da Faculdade de Odontologia de Bauru da Universidade de São Paulo ${ }^{23,25,26}$.

Baseado no Projeto Jovem Doutor, este trabalho tem como propósito desenvolver um programa de capacitação para prevenção de quedas domiciliares em idosos, uma vez que este é um tema de alta prevalência na população e acaba acarretando em inúmeras comorbidades e mortalidades. Objetiva-se também a avaliação da eficácia do programa desenvolvido neste estudo.

\section{Metodologia}

Este estudo foi desenvolvido pela Liga Acadêmica de Geriatria (LAG) da Faculdade de Medicina da Universidade de Mogi das Cruzes (FMUMC) em parceria com a Universidade Aberta à Integração (Unai), inserida na Universidade Braz Cubas (UBC), no município de Mogi das Cruzes, São Paulo.

Este projeto foi aprovado pelo Comitê de Ética e Pesquisa da Universidade de Mogi das Cruzes sob o número do parecer 1.071.034, e foi permitido o colhimento de dados na Unai. Todos os participantes assinaram o Termo de Consentimento Livre e Esclarecido (TCLE) ao aceitarem participar da pesquisa.

O projeto foi apresentado a todos os alunos da Unai. Foram selecionados 15 alunos, de ambos os sexos, com 60 anos ou mais, de forma voluntária, de acordo com o interesse e disponibilidade em participar das atividades. Este projeto foi realizado por cinco acadêmicas do curso de medicina da FMUMC, membros da LAG.

Para avaliar a eficácia do programa, foi desenvolvido um questionário contendo 16 questóes de múltipla escolha que abordavam aspectos sobre prevenção, causas e consequências das quedas. Cada questão continha quatro afirmativas, sendo apenas uma correta. Esse questionário foi aplicado aos idosos participantes antes de iniciar o programa e ao final do mesmo.

As atividades foram divididas em cinco etapas, sendo que a primeira constou da apresentaçáo do projeto para toda a sala, composta por aproximadamente 30 idosos. Entre esses, apenas 15 mostraram-se interessados em participar e assinaram o TCLE. Em seguida, foi aplicado o questionário.

$\mathrm{Na}$ segunda etapa houve apresentação da aula expositiva sobre queda domiciliar pelas acadêmicas de medicina, englobando aspectos de prevençáo, causas e consequências de quedas.
$\mathrm{Na}$ terceira etapa, após a aula expositiva, os participantes idosos foram divididos em quatro grupos. Cada grupo passou por duas estaçóes, tutoradas pelas alunas de medicina, as quais abordaram dois temas. Na primeira foi apresentado um site interativo sobre quedas domiciliares, enquanto na segunda foi exposta a Cartilha do Manual de Prevenção de Quedas. O material utilizado tanto como parte do conteúdo apresentado na aula expositiva quanto nas estaçôes tutoriais foram recursos de TIC encontrados na mídia televisiva escrita e na internet:

- Vídeo-reportagem do Bom Dia Brasil "Risco de quedas de idosos é maior dentro de casa” ${ }^{27}$ apresentado em aula expositiva;

- Manual de Prevenção de Quedas da Pessoa Idosa elaborado pelo Instituto de Assistência Médica ao Servidor Público Estadual (IAMSPE) do Hospital do Servidor Público Estadual (HSPE) ${ }^{28}$; e

- Site interativo "Casa segura para idosos" ${ }^{29}$ que aborda temas sobre prevenção de quedas na terceira idade.

Os alunos da Unai foram convidados a refletir sobre o tema e montar uma apresentaçáo acerca do assunto para disseminar o conhecimento adquirido para seus pares da Unai.

$\mathrm{Na}$ quarta etapa foram elaboradas estratégias pelos participantes da Unai em conjunto com as acadêmicas para disseminaçáo do conhecimento adquirido.

Na quinta etapa houve a reaplicação do questionário para os idosos participantes do projeto e a apresentação final dos mesmos para todos os alunos da Unai com o intuito de disseminar os conhecimentos sobre o tema abordado.

Como este trabalho foi baseado no Projeto Jovem Doutor, ao final da quinta etapa foi entregue um jaleco de agente multiplicador de conhecimento e um certificado pela participaçáo a cada idoso.

Os resultados das cinco etapas foram analisados de forma qualitativa e por meio de estatística descritiva considerando os resultados obtidos nos questionários pré e pós-capacitação.

\section{Resultados}

O projeto contou inicialmente com 15 participantes interessados, porém o número final de participantes foi de 11, devido ao fato de dois integrantes terem participado apenas do primeiro encontro. Além disso, dois outros participantes não responderam o questionário pós-capacitação integralmente e, dessa forma, tiveram que ser descartados. 
Quanto à característica epidemiológica dos participantes, $91 \%$ eram mulheres e $78,5 \%$ pertenciam à religiáo católica. Observou-se que 45,5\% eram casados, 36,4\% separados, $18,2 \%$ viúvos e $9 \%$ eram solteiros. Em relação à escolaridade, $64,28 \%$ possuíam nível superior completo.

A primeira e segunda etapas foram realizadas com apresentação do projeto e a aula expositiva sobre queda domiciliar englobando os aspectos de prevenção, causas e consequências de quedas, respectivamente, aos alunos interessados a participar, como demonstrado nas figuras $1 \mathrm{~A}$ e $1 \mathrm{~B}$.

Ainda nesse encontro, umas das participantes teve a iniciativa de criar um grupo no WhatsApp e incluir todos os integrantes da pesquisa. Esse grupo foi utilizado para esclarecer dúvidas, aproximando os participantes, e para elaborar a apresentação final. A seguir encontra-se a fala dessa participante em relação a criação do grupo: "Eu criei esse grupo com cinco universitários e 15 alunos da Unai cujo nome será Jovem Doutor".

$\mathrm{Na}$ terceira etapa, com os participantes divididos em quatro grupos, foi realizada a tutoria, utilizando os materiais interativos já mencionados. Esses foram orientados a estudar sobre o tema, sendo disponibilizado todo o material da tutoria, e incentivados a levantarem estratégias para repassarem o tema abordado a seus pares da forma que se sentissem mais confiantes (Figuras 1C e 1D).

Figura 1: A e B, aula expositiva ministrada por uma das acadêmicas da FMUMC. C e D, tutorial com os participantes divididos em quatro grupos, agosto 2016.

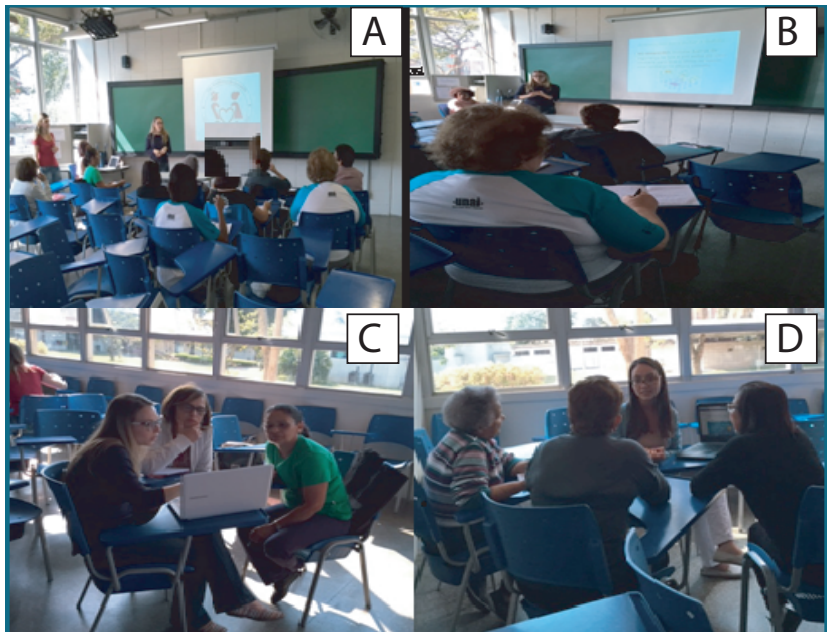

$\mathrm{Na}$ quarta etapa foram discutidas as propostas para a apresentação final. Os alunos optaram por cada grupo ficar responsável por um subtema, ficando dividido em: prevenção, causas e consequências. Cada acadêmica orientou um grupo e auxiliou na montagem da apresentação que utilizou o recurso Power Point.
Dessa forma, a organização dos encontros entre as acadêmicas e os participantes do projeto se deu através do grupo no Whatsapp, como expresso na frase por N.O.: "Terça-feira vamos nos reunir para acertarmos os detalhes. Quarta-feira apresentaremos para as meninas da Medicina. Boa noite".

$\mathrm{Na}$ quinta etapa houve a reaplicação do questionário para os idosos participantes do projeto e a apresentaçáo final para todos os alunos da Unai, disseminando conhecimento sobre o tema abordado com seus pares. O conteúdo foi exposto por meio de recurso audiovisual encontrado no Power Point, teatro e apresentação prática de objetos que podem ser utilizados na prevenção de queda domiciliar, e, ao final, os participantes receberam os jalecos intitulados "Agente Multiplicador do Conhecimento/Projeto Jovem Doutor" e um certificado de participação no projeto (Figuras 2 e 3 ).

Figura 2: Apresentação sobre prevenção de quedas domiciliares por meio de Power Point para os demais acadêmicos da Unai, setembro 2016.

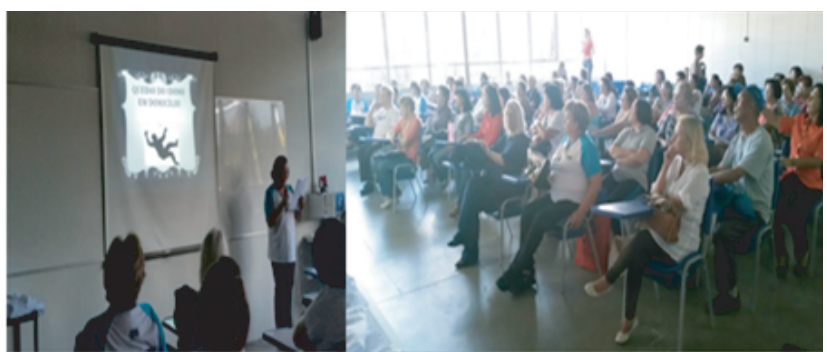

Figura 3: Participantes do projeto após a disseminação do conhecimento com os jalecos e certificados, setembro 2016.

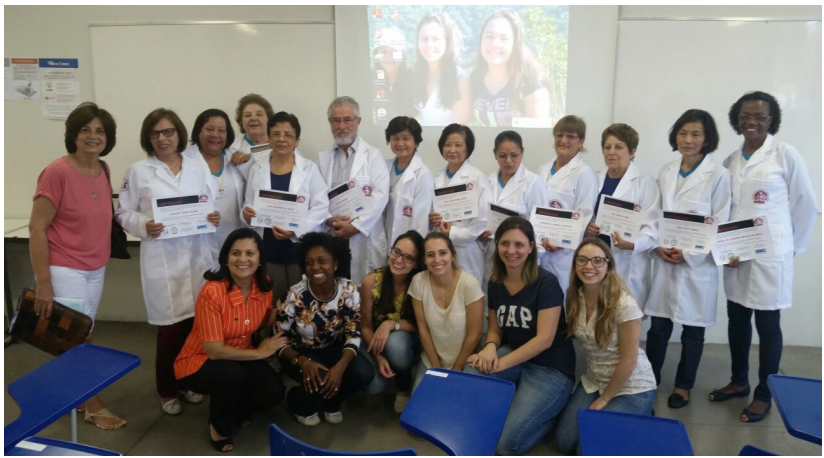

Por meio do grupo no WhatsApp, os idosos mostraram sua satisfação em participar do projeto, como consta na frase: "Obrigada pela oportunidade de aprender mais ainda sobre cuidados necessários para nossa idade. Valeu. Espero que vocês tenham muito sucesso nessa área. Quem sabe em breve podemos ser seus pacientes" (O.A.). 
Houve aumento na porcentagem de acertos a algumas questóes, no que se refere aos momentos de pré e de pós-capacitação, como mostra a figura 4.

Figura 4: Comparação entre o desempenho dos participantes idosos no questionário aplicado no pré e no pós-projeto.

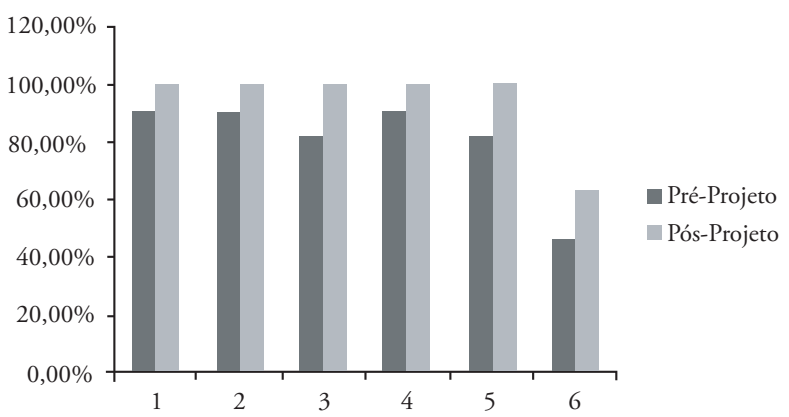

1: risco de queda na terceira idade; 2: prevenção de quedas; 3: morbimortalidade das quedas; 4: consequências das quedas; 5 : risco de quedas relacionado a bancos/cadeiras; 6: risco de quedas no banheiro.

No que se refere às questôes que abordavam quedas em escadas e qual o melhor calçado para prevenção, houve um decréscimo das porcentagens na pós-capacitação em relação à pré. No questionário de pré-capacitação, $81,81 \%$ dos participantes acertaram a questâo relacionada ao risco de ocorrência de quedas em escadas, enquanto no questionário de pós-capacitação apenas $72 \%$ dos participantes acertaram a mesma questáo. No que diz respeito ao melhor calçado para evitar a ocorrência de quedas, no questionário de pré-capacitação o índice de acerto foi de $100 \%$ dos participantes e esse número abaixou para $90,90 \%$ no questionário de pós-capacitação.

Houve manutenção das respostas em ambos os questionários, conforme ilustrado na figura 5 .

Figura 5: Questões em que os desempenhos foram os mesmos tanto no pré como no pós-projeto.

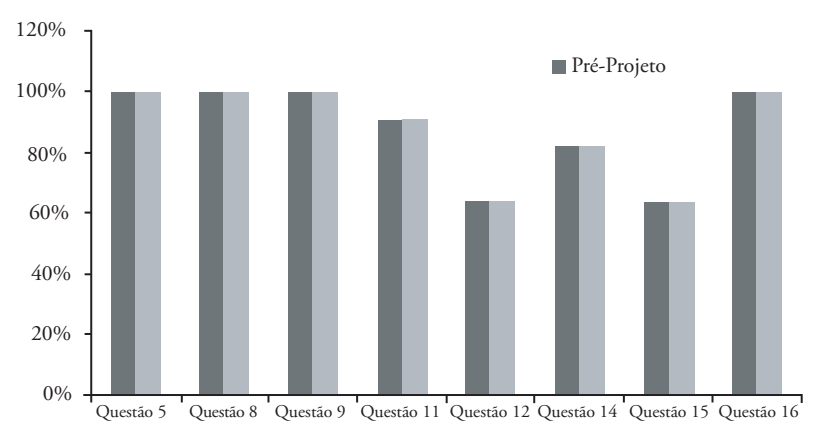

Questão 5: estratégias de prevenção de queda; Questão 8: prevenção de queda na sala de estar; Questão 9: meios de prevençấo de quedas; Questão 11: situaçôes que podem resultar em queda; Questão 12: consequências mais frequentes das quedas; Questâo 14: danos resultantes de uma queda; Questâo 15: pos sibilidade de reincidência de queda; Questáo 16: medidas de prevençáo.

\section{Discussão}

Com base nos dados disponíveis na Organização Mundial de Saúde (OMS) ${ }^{30}$, é classificado como idoso aquele que possui 60 anos ou mais nos países em desenvolvimento, como no caso do Brasil. Entre os 11 participantes do projeto a idade variava de 61 a 75 anos, sendo a maioria mulheres, o que converge com os dados obtidos na pirâmide etária divulgada pelo último censo brasileiro, no qual mostra uma representatividade das mesmas em $51 \%$ da população ${ }^{31}$.

Observou-se entre os constituintes do projeto que $45,5 \%$ eram casados, $36,4 \%$ separados, $18,2 \%$ viúvos e $9 \%$ solteiros. Com base nisso, a prevençáo nesse grupo é pertinente, uma vez que, de acordo com os resultados demostrados no estudo ${ }^{32}$, a probabilidade de óbitos por quedas em idosos aumenta significativamente para os viúvos e solteiros, ou seja, mais da metade $(54,5 \%)$ dos idosos participantes do projeto podem ter uma pré-disposição às quedas.

Pode-se observar que os idosos possuíam um bom conhecimento prévio sobre os assuntos relacionados a quedas. A maioria das questóes era conhecida por mais de $63 \%$ dos participantes, exceto quando se tratou do risco de quedas no banheiro, com apenas $45 \%$ de acerto prévio. Este dado corroborou um trabalho ${ }^{33} \mathrm{em}$ que os idosos foram entrevistados sobre o conhecimento de causas de quedas nessa faixa etária, sendo que apenas $22,5 \%$ dos participantes relataram o banheiro como um fator de risco.

Entre as questôes, $37,5 \%$ tiveram uma porcentagem maior de acertos na pós-capacitação devido ao conhecimento passado pelas acadêmicas de medicina nos encontros, enquanto $50 \%$ se mantiveram na mesma porcentagem antes e após a capacitação, e em 12,5\% houve declínio de acertos. Este declínio pode ser atribuído ao estado emocional de ansiedade dos idosos no momento de responder o último questionário, pois este foi aplicado no mesmo dia, logo antes, da apresentaçáo final para a universidade.

No estudo ${ }^{33} 56,7 \%$ da população apresentou escolaridade até a $4^{\mathrm{a}}$ série do ensino fundamental e foi verificado que os idosos conhecem parcialmente os fatores de risco. Em contrapartida, 90\% dos idosos participantes deste trabalho possuíam ensino superior completo, o que pode justificar o conhecimento prévio ou, talvez, este possa ser atribuído aos meios de acesso à informação e/ou experiências de vida da população analisada, tornando-se menos propensos a cair ${ }^{12}$.

Ainda no estudo ${ }^{33}$ foi visto que a maioria (85\%) dos idosos possui conhecimento sobre queda através da própria experiência de vida; $16,6 \%$ através da televisão, o que demonstra a escassez de informações sobre o assunto na mídia televisiva; e $0,8 \%$ através da internet. A baixa porcentagem relacionada à internet pode ser 
justificada pela dificuldade que esta população tem em lidar com a tecnologia ${ }^{33}$.

O projeto foi aplicado na Unai e pode-se observar o grande interesse que os participantes do projeto tiveram. Apesar da idade, dominavam algumas tecnologias da informação e comunicação, como WhatsApp e e-mail, sendo que tais recursos foram utilizados para a comunicação entre eles e as acadêmicas de medicina. Em uma pesquisa ${ }^{34}$ observou-se que os idosos conseguem utilizar sozinhos o Whats $A p p$ e aprendem em poucos minutos após as explicaçōes e as primeiras interações, mesmo possuindo uma dificuldade no uso de novas tecnologias. Ressalta-se que a falta de oportunidade de se aproximar da tecnologia é um dos principais motivos para que os idosos náo a utilizem.

Esse dado é contrário ao que é encontrado em algumas literaturas que mostram que o uso de produtos providos por tecnologias de interação, nesta populaçáo, não se mostra natural e fácil, apresentando-se ainda de forma conflituosa ${ }^{35,36}$ devido a sua saída do mercado de trabalho e seu menor contato com crianças ${ }^{37}$.

Outros estudos mostram que para fazer parte da atual sociedade inclui se adentrar no processo tecnológico desta, visto que a tecnologia está em todos os lugares: aparelhos eletrodomésticos, caixas eletrônicos, supermercados, aparelhos celulares, entre muitos outros $^{38}$. O idoso que está inserido no processo tecnológico sente-se menos excluído da sociedade e acaba por melhorar seu contato com o mundo exterior ${ }^{38,39}$. Dessa forma, observa-se a importância de pensar em estratégias para promover a inclusão digital da terceira idade ${ }^{38}$.

Ficou notória a rápida aceitação dos participantes da Unai em relação às tecnologias utilizadas para expor as informaçôes que seriam passadas, não oferecendo resistência a nenhum dos recursos e se utilizando deles para os estudos posteriores, domiciliares. Vale ressaltar que a aceitação dos participantes pelos recursos de Telessaúde ocorre devido a um distanciamento do vocabulário técnico e maior confiança quanto à veracidade das informaçóes passadas ${ }^{40}$.

\section{Conclusão}

Foi realizado o programa de prevençáo de quedas domiciliares e constatou-se que os idosos participantes demonstraram grande interesse pelo tema e pelas TICs.

O programa mostrou-se eficaz uma vez que houve extensão dos conhecimentos prévios sobre o tema, visto através do aumento no índice de acertos no questionário de pós-capacitação em comparação ao questionário de pré-capacitação em parte das questóes.
Houve êxito na disseminaçáo do conhecimento com o uso das TICs e outras estratégias por parte dos participantes aos demais alunos da Unai.

A importância de um projeto dessa natureza é fazer que os conhecimentos adquiridos dentro da universidade possam extravasar seus muros e sejam úteis à comunidade que os cerca, atuando de maneira a promover a saúde e a prevenção de doenças.

\section{Referências}

1. Rezende CP, Gaede-Carrillo MRG, Sebastião ECO. Queda entre idosos no Brasil e sua relação com o uso de medicamentos: revisão sistemática. Cad Saúde Públ. [citado em 2016 jun. 23]. Disponível em: http:// www.scielosp.org/scielo.php?script=sci_arttext\&pi$\mathrm{d}=$ S0102-311X2012001400002\&lng=en. http://dx.doi. org/10.1590/S0102-311X2012001400002.

2. Instituto Brasileiro de Geografia e Estatística [homepage na internet]. Expectativa de vida. 2013. [citado em 2014 maio 1]. Disponível em: http://teen.ibge.gov.br/ noticias-teen/7827-expectativa-de-vida.2.

3. Instituto Brasileiro de Geografia e Estatística [homepage na internet]. Perfil dos idosos responsáveis pelos domicílios no Brasil: 2002 [citado em 2014 jun 27]. Disponível em: http:// www.ibge.gov.br/home/presidencia/noticias/25072002pidoso.shtm

4. Galera SC, Almeida MM, Gabrielle RR, Aragão LP, Freitas LRS, Freire Neto JB. Estágio supervisionado em Atenção à Saúde do Idoso do Curso de Medicina da Universidade de Fortaleza. Abmes Cad. 2011;21:35-454.

5. Brasil VJW, Batista NA. O Ensino de Geriatria e Gerontologia na Graduação Médica. Rev Bras de Ed Méd. 2015;39(3):344-51.5.

6. Costa NRCD, de Aguiar, MIF, Rolim ILTP, Rabelo PPC, Oliveira DLA, Barbosa YC. Política de saúde do idoso: percepção dos profissionais sobre sua implementação na atenção básica/health policy for elderly people: perception of professional about its implementation in primary care. Rev de Pesq em Saúde. 2016;16(2).6.

7. Pereira AMVB, Schneider RH, Schwanke CHA. Geriatria, uma especialidade centenária. Sci. med. 2009 out-dez; 19(4):154-61.

8. Anjos CB, Ferreira MCG, Ferreira MA. Política Nacional do Idoso e sua implementaçáo na assistência de enfermagem. Raízes e Rumos. 2014; 2(1):3-11.8.

9. Santos DA, Belo MCF, Sena KL, Mota GBC. Análise comparativa da capacidade funcional de idosas com e sem incontinência urinária. Rev Fisio Bras. 2012 nov-dez; 13(6):118-23.

10. Moraes EN, Marino MCA, Santos RR. Principais síndromes geriátricas. Rev Med Minas Gerais. 2010; 20(1): 54-66.

11. Inácio CCF. Prevençáo de queda em idosos: possibilidades da garantia de um envelhecimento saudável. Salvador. 
Monografia [Titulo de Especialista de Enfermagem em Emergência] - Universidade Castelo Branco; 2011.

12. Pereira GN, Morsch P, Lopes DGC, Trevisan MD, Ribeiro A, Navarro JHN, et al. Fatores socioambientais associados à ocorrência de quedas em idosos. Ciênc Saúde Col. [citado em 2016 jun. 23]. Disponível em: http://www.scielo.br/scielo.php?pi$\mathrm{d}=$ S1413-81232013001200007\&script $=$ sci_abstract\&tlng=pt

13. Landim ACF, Pinheiro FM, Pessanha FS, Santos L, Valente GSC. Assistência de enfermagem a idosos com traumas ósseos: uma revisão integrativa. J Res Fundam Care Online. 2015; jan-mar; 7(1):2083-103.

14. Cruz DT, Cruz FM, Ribeiro AL, Veiga CL, Leite ICG. Associaçáo entre capacidade cognitiva e ocorrência de quedas em idosos. Cad Saúde Colet. [citado em 2016 out. 3] Disponível em: http://www.scielo.br/scielo.php?script=sci_arttext\&pid=S1414462X2015000400386\&lng=pt.14.

15. Freitas R, Santos SSC, Hammerschmidt KSA, Silva ME, Pelzer MT. Cuidado de enfermagem para prevenção de quedas em idosos: proposta para ação. Rev Bras Enferm. [citado em 2016 jun. 23] Disponível em: http://www.scielo.br/scielo. php?script=sci_arttext\&pid=S0034-71672011000300011.

16. Oliveira AS, Trevizan PF, Bestetti MLT, Melo RC. Fatores ambientais e risco de quedas em idosos: revisão sistemática. Rev Bras Geriatr Gerontol. [citado em 2016 out. 3]. Disponível em: http://www.scielo.br/scielo.php?script=sci_arttext\&pid=S1809-98232014000300637\&lng=pt.

17. Cruz DT, Ribeiro LC, Vieira MT, Teixeira MTB, Bastos RR, Leite ICG. Prevalência de quedas e fatores associados em idosos. Rev Saúde Públ. [citado em 2016 jun. 23]. Disponível em: http://www.scielo.br/scielo.php?script=sci_arttext\&pid=S0034-89102012000100017\&lng=en.

18. Ansai JH, Glisoi SFN, Oliveira T, Soares AT, Cabral KN, Sera CTN et al. Revisão de dois instrumentos clínicos de avaliação para predizer risco de quedas em idosos. Rev Bras Geriatr Gerontol. [citado em 2016 out. 1]. Disponível em: http://www.scielo.br/scielo.php?script=sci_arttext\&pi$\mathrm{d}=$ S1809-98232014000100177.18.

19. Gontijo KCP. Proposta de intervenção na prevenção de quedas dos idosos no ambiente familiar. Minas Gerais. Monografia [Curso de Especialização em Saúde da Família] - Universidade Federal de Minas Gerais; 2011. Disponível em: www.nescon. medicina.ufmg.br/biblioteca/imagem/3129.pdf19.

20. Maia BC, Viana PS, Arantes PMM, Alencar MA. Consequências das quedas em idosos vivendo na comunidade. Rev Bras Geriatr Gerontol. [citado em 2016 out. 1] Disponível em: http://www.scielo.br/scielo. php?script=sci_arttext\&pid=S1809-98232011000200017.

21. Antes DL, Schneider IJC, d'Orsi E. Mortalidade por queda em idosos: estudo de série temporal. Rev Bras Geriatr Gerontol. [citado em 2016 out. 3]. Disponível em: http://www.scielo.br/pdf/rbgg/v18n4/pt_1809-9823-rbgg-18-04-00769.pdf. 21.

22. Pinho TAM, Silva AO, Tura LFR, Moreira MASP, Gurgel $\mathrm{SN}$, Smith AAF et al. Avaliação do risco de quedas em idosos atendidos em Unidade Básica de Saúde. Rev Esc Enferm USP. [citado em 2016 out. 1]. Disponível em: http://www.scielo.br/scielo.php?script=sci_arttext\&pi$\mathrm{d}=$ S0080-62342012000200008.22.

23. Picolini MM. Teleducação interativa na capacitação de estudantes do ensino fundamental em síndromes genéticas. [Dissertação de Mestrado]. São Paulo: Departamento de Fonaudiologia da Faculdade de Odontologia de Bauru da Universidade de São Paulo; 2011.

24. Macéa DD, Rondon S, Chaar LJ, Wen CL. Public health education for young students aided by technology. J Telemed Telecare. 2009 apr;15 (3):159.

25. Blasca WQ, Picolini MM, Silva ASC, Campos K, Pinto GFR, Brasolotto AG, et al. Projeto Jovem Doutor Bauru: capacitaçáo de estudantes do ensino médio em saúde auditiva. Rev Cefac. [citado em 2016 jun. 23]. Disponível em: http://www.scielo.br/pdf/rcefac/2013nahead/189-11.pdf.

26. Blasca WQ, Corrêa CC, Picolini MM, Campos K, Silva ASC, Berretin-Félix G, Brasolotto AG, Maximino LP. Una estrategia de teleducación sobre lasalud auditiva y vocal en Brasil. Rev Logop Foniatr Audiol. 2015 jan- mar; 35(1):2-7.

27. Rede Globo. Risco de Quedas de Idosos é maior dentro de casa. Jornal Bom Dia Brasil [Reportagem]. São Paulo: Rede Globo; 2012 [citado em 2014 dez. 3]. Disponível em: http//:globotv.globo.com/rede-globo/bom-dia-brasil/v/risco-de-quedas-de-idosos-e-maior-dentro-de-casa/2060964/.

28. IAMSPE. Manual de prevenção de quedas [manual em pdf]. São Paulo: VFR Serviços de Comunicação; 2014.

29. Martins C. Casa segura para idosos - Adaptaçôes em casa ajudam a garantir velhice saudável [Internet]. São Paulo; 2011 [citado em 2014 dez. 4]. Disponível em: http://delas. ig.com.br/casa/arquitetura/adaptacoes-em-casa-ajudam-a-garantir-velhice-saudavel/n1237726491017.html.

30. Organização Mundial da Saúde (OMS). Mulheres e Saúde: evidências de hoje, agenda de amanhã. 2011. [citado em 2014 maio 27]. Disponível em: www.who.int/ageing/mulheres_saude.pdf. 30 .

31. Instituto Brasileiro de Geografia e Estatística [homepage na internet]. Censo 2010: População Homens x Mulheres [citado em 2016 jul. 27]. Disponível em: http:// www.censo2010.ibge.gov.br/sinopse/webservice/default. php? $\operatorname{cod} 1=0 \& \operatorname{cod} 2=\& \operatorname{cod} 3=\&$ frm $=$ hom_mul

32. Rosa TSM, Moraes AB, Peripolli A, Santos Filha VAV. Perfil epidemiológico de idosos que foram a óbito por queda no Rio Grande do Sul. Rev Bras Geriatr Gerontol. Rio de Janeiro, 2015; 18(1):59-69.

33. Silva NSM, Lopes AR, Mazzer LP, Trelha CS. Conhecimento sobre fatores de risco de quedas e fontes de informação utilizadas por idosos de Londrina (PR). Rev Kairós Gerontol. 2014 Jun;17(2):141-51.

34. Lima DS, José HSS, Ferreira SBL, Capelli C, Santos G, Santoro FM. Avaliação da Primeira Experiência de Uso do Aplicativo WhatsApp por Usuários da Terceira Idade. Rio de Janeiro, 2015;77-84 
35. Fagundes VH, Santos AS. As tecnologias de interação e as relaçóes de usol pela terceira idade: um estudo de caso no segmento de linha branca. Blu Des Proc. 2015;2(2):1251-61.

36. Bianchetti L. Da chave de fenda ao laptop. Tecnologia digital e novas qualificaçóes: desafios à educação. 2. ed. Florianópolis: Editora da UFSC; 2008.

37. Rosen LD, Weil MM. Adult and teenage use of consumer, business, and entertainment technology: potholes on the information superhighway?. J of Cons Affairs. 1995; 29(1): 55-84.
38. Tavares MMK, Souza STC. Os idosos e as barreiras de acesso às novas tecnologias da informação e comunicação. Rev Ren. Rio Grande do Sul; julho 2012;10(1).

39. Morris JM. Computer training needs of older adults. Educational Gerontology. 1994;20(6): 541-55.

40. Huis in 't Veld RM, Widya IA, Bults RGA, Sandsjö L, Hermens HJ, Vollenbroek-Hutten MM. A scenario guideline for designing new teletreatments: a multidisciplinary approach. J Telemed Telecare. 2010;16(6): 302-7.

Como citar este artigo:

Campos K, Santos MA, Barros NM, Simionato TM, Brandão JGP, Ramos APMC. Capacitação de idosos na prevençáo de quedas domiciliares utilizando tecnologias da informação e comunicação. Rev. Aten. Saúde. 2017;15(51):84-91. 